\title{
Revisión literaria e histórica de medidas de complejidad de proyectos $y$ desempeño de proyectos
}

\section{Héctor Julio Polo Ábrego ${ }^{1, *}$, Yarissa M. Palma ${ }^{2}$}

${ }^{1}$ Estudiante de doctorado de la Universidad Tecnológica de Panamá (UTP), Dirección de Postgrado, Ingeniería de Proyectos

${ }^{2}$ Universidad Tecnología de Panamá, Dirección de Gestión y Transferencia del Conocimiento

*Autor para correspondencia. Email: hjpoloa@gmail.com

Recibido: 26 de septiembre de 2020

Aceptado: 08 de octubre de 2020

\section{Resumen}

Esta revisión literaria es una indagación acerca de metodologías de medición del desempeño y complejidad de los proyectos, que evidencia la no existencia de una comparación entre ambas mediciones bajo la definición estándar de desempeño. Se establece la diferencia entre las metodologías holísticas o cualitativas, y aquellas basadas en la teoría de grafos aplicada, y se exploran las variaciones y tendencias actuales de mediciones del desempeño y el éxito de los proyectos, llevándonos a reevaluar definiciones de los conceptos investigados y apreciar las ventajas que resultan en utilizar los métodos basados en la teoría de grafos aplicada. Se concluye que los cronogramas de obra pueden, de estar bien realizados, ser utilizados como base para la medición de la complejidad de proyectos.

Palabras Clave: Complejidad de proyectos, valor ganado, índice de complejidad, índice de desempeño del cronograma

\begin{abstract}
This literary review seeks methodologies to measure the performance and complexity of projects, and to demonstrate the non-existence of a comparison between both measurements under the standard definition of performance. The difference between qualitative and holistic methodologies and those based on applied graph theory is established, and current variations and trends in project performance and success measurements are explored, leading us to reevaluate the investigated concepts' definitions and appreciate the advantages that result from using methods based on applied graph theory. It is concluded that work schedules can, done properly, be used as a basis for measuring project complexity.
\end{abstract}

Keywords: Project complexity, earned value, complexity index, schedule performance index. 


\section{Estado del Arte - Introducción}

La intención de esta primera sección es la de explorar la literatura más reciente con tal de esclarecer los conceptos de: proyecto, complejidad, la complejidad de un proyecto y el desempeño de un proyecto, valor agregado, y el concepto del éxito de un proyecto también será explorado.

Seguido a la aclaración de estos conceptos, se abordan las dos grandes vertientes en el análisis de la complejidad de un proyecto, la que modela elementos de forma holística de un proyecto, y la que modela estrictamente los elementos finitos de los cronogramas de proyectos.

De acuerdo con esta premisa, se establecerá una base teórica para adentrarnos en el método especifico utilizado en los cálculos de esta tesis.

\subsection{Definiciones de Proyecto, Complejidad, Desempeño y Éxito}

\subsubsection{Proyecto}

Un proyecto se define, según el PMBOK (Project Management Book of Knowledge o el Libro de Conocimientos de la Administración de Proyectos del Project Management Institute, PMI, el Instituto de Administración de Proyectos), como "el esfuerzo que se lleva a cabo para crear un producto, servicio o resultado único, y tiene la característica de ser temporal. No es ni el producto, servicio o resultado único por lo cual se hacen estos esfuerzos” [1]. pg. 5. Esta es una definición ya aceptada por la industria y será base de nuestra investigación.

\subsubsection{Complejidad}

Existe un consenso de que la definición de "complejidad", y la misma aplicada a proyectos, ha sido difícil de determinar. En esta sección llegaremos a explorar varias propuestas de autores que han abarcado el tema orientándolo a la administración de proyectos.

\subsubsection{Propuesta de la definición de complejidad por Baccarini, 1996}

Baccarini explora varios acercamientos al concepto de complejidad por otros autores. Inicia con el concepto de complejidad como una "dimensión del proyecto en sî" [2]. Continua con la definición de diccionario en la cual se define como aquello que consiste en muchas partes interrelacionadas [2]. De aquí deriva el concepto de la diferenciación de los elementos y la interconectividad de estos, comentando que "esta interpretación de complejidad refleja la teoría de sistemas en la que un sistema complejo es frecuentemente definido en términos de diferenciación y conectividad" [2] 
Finalmente, su propuesta para la definición de la complejidad de un proyecto es la siguiente:

"Se propone que la complejidad del proyecto 'consta de muchas partes interrelacionadas y variadas' y que se pueda operacionalizar en términos de diferenciación e interdependencia." [2]

Esta definición es posteriormente aceptada por Wood, 2010 [3]

\subsubsection{Las cuatro asociaciones de complejidad por Frame, 2002}

J. Davidson Frame en el 2002 estudia el problema de los Proyectos complejos iniciando con el concepto de "complejidad". No logra hacer una definición puntual, sin embargo, la asocia con 4 características: el tamaño, la variedad, la dificultad y el cambio. De seguido abordaremos estos cuatro aspectos bajo la perspectiva de Frame.

\section{a. La complejidad se asocia con el tamaño del objeto}

Frame afirma que "generalmente, los objetos grandes con muchos componentes tienden a ser más complejos que aquellos más pequeños con menos componentes” pg.25. [4] Igualmente desarrolla la fórmula del máximo número de relaciones entre estos elementos: "n(n-1)/2, o (n2n) /2" pg.25[4], la cual veremos que será utilizada como base de la fórmula de complejidad de cronogramas desarrollado por Naga y Hageb.

\section{b. La complejidad se asocia con la variedad}

Se refiere a la cantidad de opciones existentes a la hora de tomar todo tipo de decisiones. "El dilema de las opciones excesivas que un gerente de proyecto y su equipo se enfrentan es obvio" pg.27[4]. También menciona que las particularidades de un proyecto generalmente no se repiten con otro: "el hecho de que sus proyectos tienden a ser únicos (a los gerentes de proyectos) los confunde porque las lecciones aprendidas de "sortear" por opciones de su proyecto anterior son frecuentemente irrelevantes en el [proyecto] actual" pg.27 [4].

\section{c. La complejidad se asocia con la dificultad}

"Cuando algo es difícil de realizar es generalmente visto como más complejo que algo que es fácil” pg. 28. Aquí el autor hace comparaciones con el cálculo y la aritmética, siendo la primera más compleja que la segunda. Sin embargo, agrega que "la complejidad enraizada en la dificultad es generalmente manejada a través de la maestría," que se traduce en "educación y experiencia" pg.28 [4]

\section{d. La complejidad se asocia con el cambio}

"La rapidez del cambio en el mundo de hoy contribuye hasta cierto grado a la complejidad. Una manera en que lo hace es creando un blanco en movimiento". pg. 30 [4] Bajo este concepto Frame incluye temas como cambios tecnológicos, aumento exponencial en la información disponible, en los cambios en los alcances y requerimientos de los proyectos, entre otros [4] 


\subsubsection{El acercamiento dual de Vidal sobre el concepto de complejidad de proyectos, 2007}

Vidal, et. Al., tiene dos conceptos sobre complejidad. Uno que desarrolla dentro de su metodología posterior, que es la complejidad de la administración misma del proyecto, y la complejidad sistemática del mismo.

La primera la define como "la cadena de decisiones generales" tomadas. [5]

La complejidad sistemática lo define analógicamente con un ejemplo que parte de la intuición y no necesariamente proporcione algún resultado.

Vidal dice que esta complejidad sistemática "puede entenderse fácilmente a través de un ejemplo simple, el del nivel de detalle de la estructura de desglose del trabajo. Si la estructura de desglose del trabajo no es lo suficientemente extensa, el proyecto puede haberse descompuesto débilmente y los paquetes de trabajo no se han definido correctamente: como consecuencia, los paquetes de trabajo podrían descomponerse en subtareas que no ban sido definidas por el gerente del proyecto y es probable que la autoorganización resultante de esta ausencia de detalles afecte mal el desempeño del proyecto. Por el contrario, si la estructura de desglose del trabajo es demasiado extensa, la descomposición puede ser demasiado compleja para usarse de manera efectiva, ya que las interacciones y las interrelaciones entre los paquetes de trabajo pueden ser demasiado numerosas para ser gestionadas adecuadamente." [5]

Vidal nos muestra una gráfica teórica de la relación entre la complejidad de un proyecto y el desempeño del mismo.

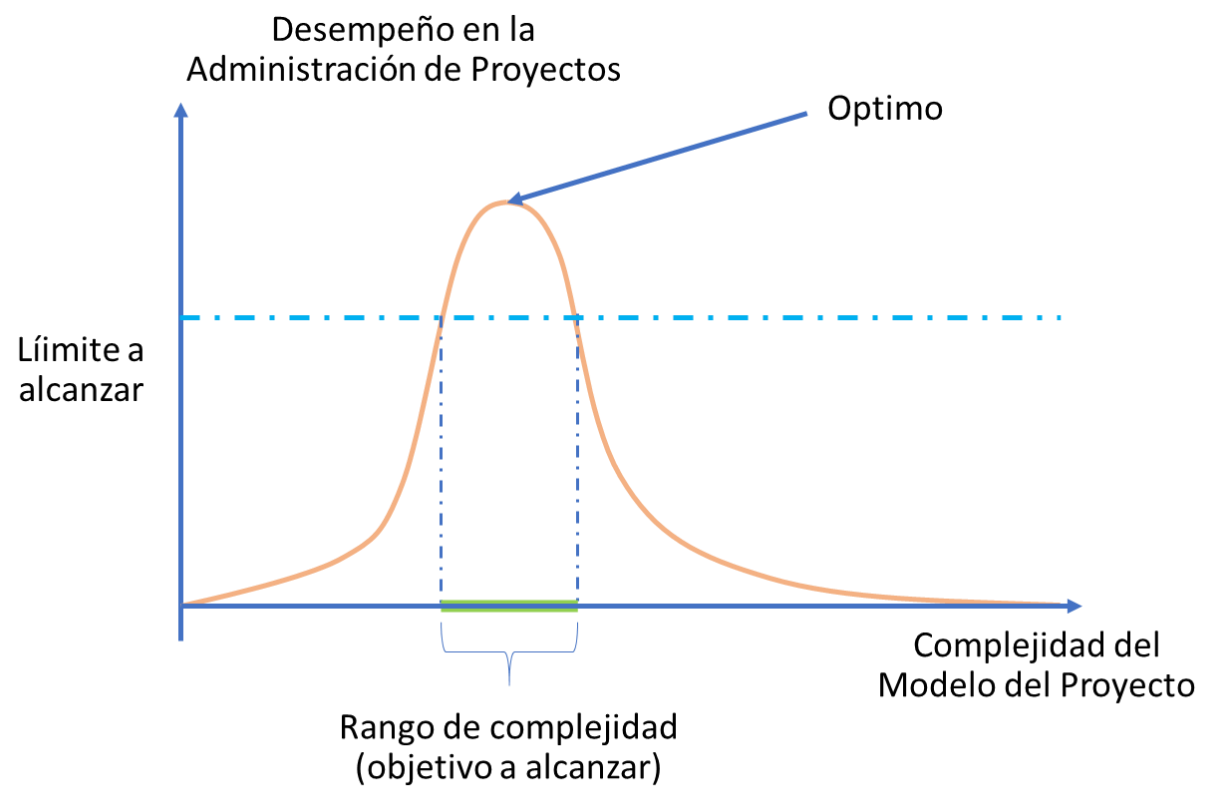

Ilustración 1 - Curva de complejidad optima [5] 
En esta grafica se puede observar que, según la interpretación de Vidal, debe haber un nivel óptimo de complejidad que permita desarrollar el proyecto con buen desempeño. Muy poca complejidad no permite alcanzar el máximo desempeño, y demasiada complejidad tampoco.

Esta teoría de Vidal está basada en un concepto de estructura de trabajo, que es en esencia, como mencionan Nassar y Hageb, la complejidad del cronograma y no necesariamente del proyecto en sí. [6]

Sin embargo, Vidal en un artículo posterior del 2011, menciona 4 aspectos que luego retomaremos en la exploración de la definición de la complejidad de proyectos. Estos 4 aspectos son: "1. el aspecto teleológico, 2. el aspecto funcional 3. el aspecto ontológico y 4. el aspecto genético" [7].

\subsubsection{Teoría de la complejidad como fenómeno social de Weaver, 2009}

Weaver, considera que la teoría de complejidad tiene que ver con las relaciones sociales dentro del proyecto, por encima de cualquier otro aspecto: "La esencia de CRPR ("Complex Responsive Processes of Relating" o Procesos Receptivos Complejos del Relacionarse) y la teoría de redes sociales es que el resultado final del proyecto, su éxito o fracaso, es creado por miles de decisiones individuales tomadas por los miembros del equipo del proyecto en el curso de sus interacciones diarias entre sí, a través de conversaciones, incrustadas en sus relaciones. Es imposible que el Gerente de Proyecto esté al tanto de cada conversación y decisión". [8]

Donde CRPR se define como: "Los procesos de respuesta complejos (CRPR) ponen énfasis en la interacción entre las personas y la naturaleza esencialmente receptiva y participativa de los procesos humanos de organización y relación. La organización es una propiedad emergente de muchos seres humanos individuales que interactúan juntos a través de sus complejos procesos receptivos de relacionarse. (Stacey, Griffin, Shaw. 2000) Utilizan el lenguaje en las conversaciones para transferir simultáneamente información e ideas, negociar el estatus social y desarrollar relaciones de poder (Cooke-Davies, et al. 2007)”. [8]

Para Weaver, el tema de la complejidad se define bajo este concepto de relaciones sociales, pero sigue siendo solo un elemento de las dimensiones a tomar en cuenta a la hora de planificar y controlar un proyecto utilizando los cronogramas de obra. Esto lo evidencia en sus 4 puntos describiendo las dimensiones de un proyecto:

"1. Su tamaño inherente generalmente medido en términos de valor; 2. El grado de dificultad técnica para crear la salida; 3. El grado de incertidumbre involucrado en el proyecto; y 4. La complejidad de las relaciones ("pequeña p" política) tanto dentro del equipo del proyecto como en torno al proyecto ". [8]

Interesantemente, para Weaver, recae en el "cronogramista" o planificador la responsabilidad de atender sobre todo las dificultades técnicas del cronograma y a su vez estar al tanto y trabajar en el punto de la complejidad "social" del proyecto, en conjunto con el gerente del proyecto. [7] 


\subsubsection{Complejidad de un proyecto por Luo, Lan et. Al., 2012}

Luo presenta 3 claros acercamientos, los primeros dos para llegar a la definición de complejidad, y luego el tercero para definir la complejidad de un proyecto. Menciona que "la complejidad es definida al tener un numero grande de partes interactuando" [9] y que "la ciencia de la complejidad es el estudio de estas interacciones." [9]. Por ende, para Luo, la complejidad de un proyecto es "considerada una de las más fundamentales propiedades de un proyecto y resulta de la interacción de sus diferentes partes con propiedades estructurales, dinámicas e inciertas" [9]

\subsection{El concepto de desempeño de proyectos y su diferencia con el éxito del proyecto.}

\subsubsection{Medición de desempeño utilizando el método de valor ganado.}

El PMI le llama desempeño del proyecto al cociente resultante de dividir el valor ganado, entre el valor planificado, en algún momento del tiempo del proyecto. A este resultado le llama el índice de desempeño del cronograma. Las variables son las siguientes:

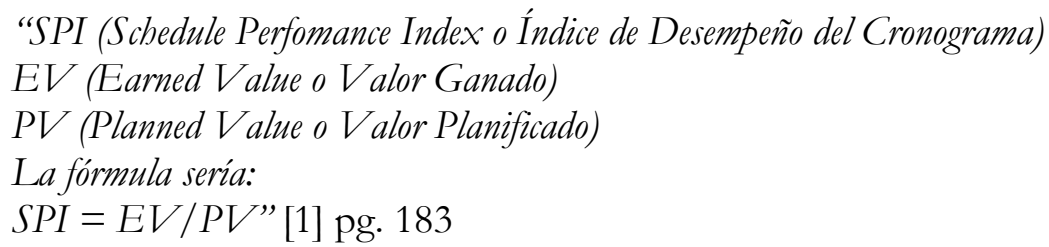

Esta fórmula adoptada por el PMI fue desarrollada inicialmente por el cuerpo de defensa de los Estados Unidos [4, 10]. En sí, mide el avance económico permitiendo observar desde el inicio problemas que pueden resultar a la larga en un proyecto impactado. Su enfoque en costos no quiere decir que no tome en cuenta el factor tiempo, ya que el mismo se basa en utilizar las duraciones de las actividades del proyecto para crear el comportamiento base del plan del proyecto, y compararlo con las cifras reales, tanto de valor, como de costos.

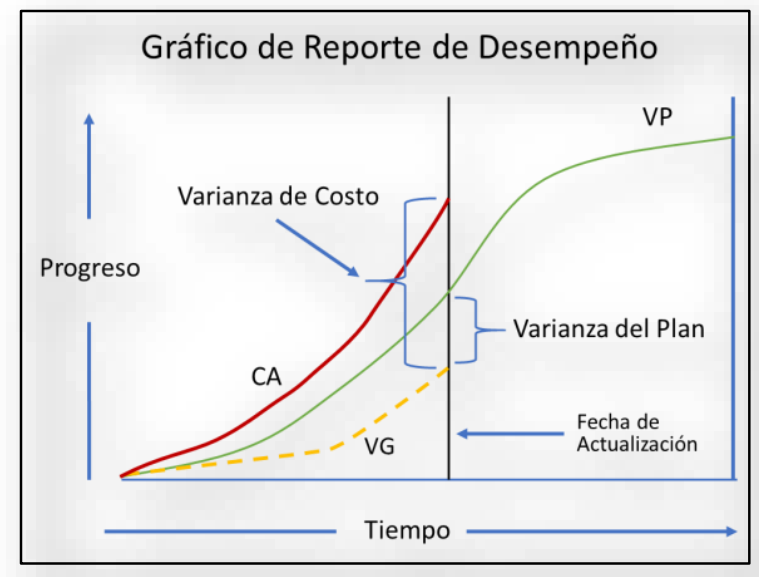

Ilustración 2 - Gráfica Ejemplo de Valor Ganado [11] 
Lo que no se puede ver con una curva general de todo el proyecto, se podría ver en análisis realizados a secciones de estos, de ser un proyecto con mayor complejidad y tamaño.

\subsubsection{Distinción del desempeño y el éxito de un proyecto}

Luo (2012), expresa la definición del desempeño del proyecto como aquello que "principalmente se enfoca en los indicadores de la ejecución del proyecto, mientras que el éxito del proyecto incluye el periodo posterior a su terminación y su influencia" [9]

Luo menciona que la definición de éxito del proyecto "consistía en cumplir con el triángulo dorado de tiempo, costo y calidad" [9], pero considera esta definición como el "criterio original de medir el éxito de un proyecto, y que, aunque son importantes, no incluyen medidas de factores modernos del éxito de un proyecto." [9]. Entre estos factores, menciona el "tiempo, la calidad, la salud y seguridad, el rendimiento ambiental, la satisfacción de los participantes, la satisfacción del usuario, y el valor comercial." [9]

\subsection{Complejidad de Proyectos - Vertiente holística}

La palabra holística según la real academia de la lengua española se define como:

"Que pertenece a... (ver holismo) una doctrina que propugna la concepción de cada realidad como un todo distinto de la suma de las partes que lo componen." [12]

La complejidad de proyectos ha sido observada de manera holística, es decir, como aquel concepto que, al lograr discernirla de sus partes que la componen, nos puede proveer de una visión más clara de lo que representa.

Con una analogía sencilla, el ser humano es en sí la suma de todas sus partes: manos, piernas, cabeza, corazón, espíritu. Sin embargo, el ser humano no es sus manos, no es sus piernas, ni su corazón ni su espíritu.

La complejidad de proyectos ha sido observada de manera holística en la mayor parte de la literatura disponible sobre la gestión de proyectos [3,5,7,8,13,14,15-21]. En esta manera holística/cualitativa de ver la complejidad de proyectos es preciso sopesar o ponderar los factores cualitativos para tratar de cuantificar elementos que, de otra manera, serían muy difíciles de medir [7]. En estos casos, el investigador se ve en la difícil tarea de proponer un método estadístico que refleje el consenso y el aval de opiniones de varios profesionales $[7,15]$.

Para identificarlos dentro de esta categoría, he tomado los siguientes aspectos para determinar el criterio de clasificación:

1. En su mayoría los elementos son cualitativos o difíciles de cuantificar, y deben pasar por un proceso de ponderación para su cuantificación.

2. Contienen elementos fuera del proyecto en sí (véase sección 1.1.1. Proyecto de este artículo sobre la definición de un proyecto).

3. Requieren de una revisión y aprobación posterior por parte de los participantes de los métodos propuestos. 
Dentro de estos elementos o factores se encuentran temas como: el análisis del cliente, estructura organizacional (roles, canales de comunicación, comunicación regular, la experiencia del equipo, la calidad de la toma de decisiones), resultado de arbitrajes, expectativas de los accionistas o involucrados, el tamaño del proyecto, las interdependencias del proyecto con el ambiente, la interdependencia de los departamentos, la interdependencia de los sistemas de información, el contexto cultural, y la complejidad tecnológica, entre otras $[7,13]$.

El resultado de estos acercamientos son los diferentes métodos propuestos como lo son el método analítico por jerarquía [7] y la utilización del método Delphi [14]. Otros acercamientos se limitan al modelado como una herramienta de análisis dentro de los procesos administrativos [13], o proponen una cuantificación de procesos meta-proyectuales (más allá del análisis de un solo proyecto) $[7,15]$.

En la siguiente sección se exploran las metodologías resultantes debido a las conclusiones tomadas por varios autores dentro de esta clasificación holística de la complejidad de proyectos.

\subsubsection{Complejidad de Proyecto utilizando el método de descomposición funcional/dimensional, Sinha, 2006 [22]}

Sinha propone utilizar el sistema de descomposición funcional para evaluar la complejidad de las actividades del proyecto. Asume que "que la complejidad de una actividad de proyecto depende del número de sus dimensiones y los niveles de sus factores generadores de complejidad." De este concepto desarrolla la siguiente formula:

$$
C I=N+\sum_{i=1}^{L} C F G_{i} L
$$

En donde "Donde $\mathrm{N}$ es el número de dimensiones de una actividad de proyecto, CGFi es el número de factores generadores de complejidad en el nivel i y L es el número de nivel.” [22]

La razón por la cual se incluye dentro del grupo de definiciones holísticas de complejidad, yace en los factores que Sinha incluye dentro de su descomposición funcional/dimensional.

Estos factores se pueden apreciar en su análisis ejemplo representado por un modelo jerárquico de dimensiones recreada en la siguiente imagen: 


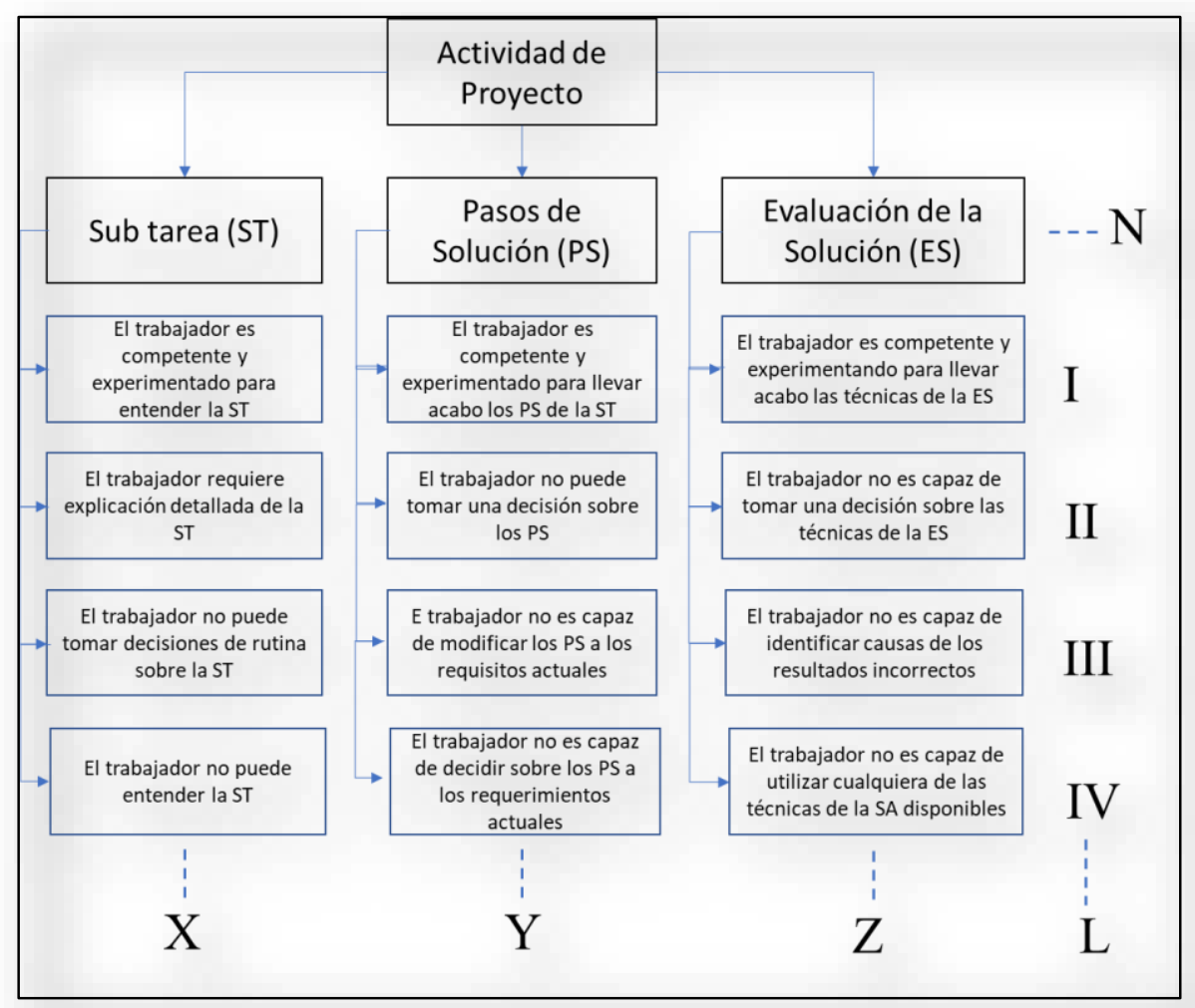

Ilustración 3 - Descomposición dimensional de una actividad de proyecto [22]

Estos elementos presentados requieren de una apreciación del gerente del proyecto. Sinha menciona que para esta evaluación "las actividades del proyecto fueron de un proyecto de construcción completado anteriormente y fueron administradas por un gerente de proyecto con 30 años de experiencia." [22] La tarea de realizar esta evaluación recae sobre el equipo

administrativo, o como lo menciona Sinha, sobre el "gerente de proyecto" [22] agregando elementos cualitativos dentro del análisis.

\subsubsection{Complejidad vista desde un punto de vista de modelado de categorías, Vidal, 2007 [5]}

Seguida tabla replicada de su categorización de las aristas o elementos incluidos dentro de su modelado. 


\begin{tabular}{c|c|c|c|}
\hline \multicolumn{5}{|c|}{ E1 Marco de Referencia (ALOE) } \\
\hline Atributos & Enlaces & Objetos & Eventos \\
\hline Calidad & Enlace jerárquico & Objetivo & Decisión interna \\
\hline Costo & Enlace contributivo & Entregable & Decisión externa \\
\hline Duración, fecha de inicio & Enlace secuencial & Actividad & Riesgo interno \\
\hline Progreso & Enlace de influencia & Recurso & Riesgo externo \\
\hline Descripción & Enlace de cambio & $\begin{array}{c}\text { Otro proyecto dentro de } \\
\text { la firma }\end{array}$ & \\
\hline Recursos asignados & & & \\
\hline Valor agregado & & & \\
\hline
\end{tabular}

Tabla 1 - Marco de referencia ALOE (Atributos, Enlaces (Links), Objetos, Eventos) [5]

El resultado buscado por Vidal es una representación visual que ayuda a la comunicación de los involucrados en el manejo del proyecto.

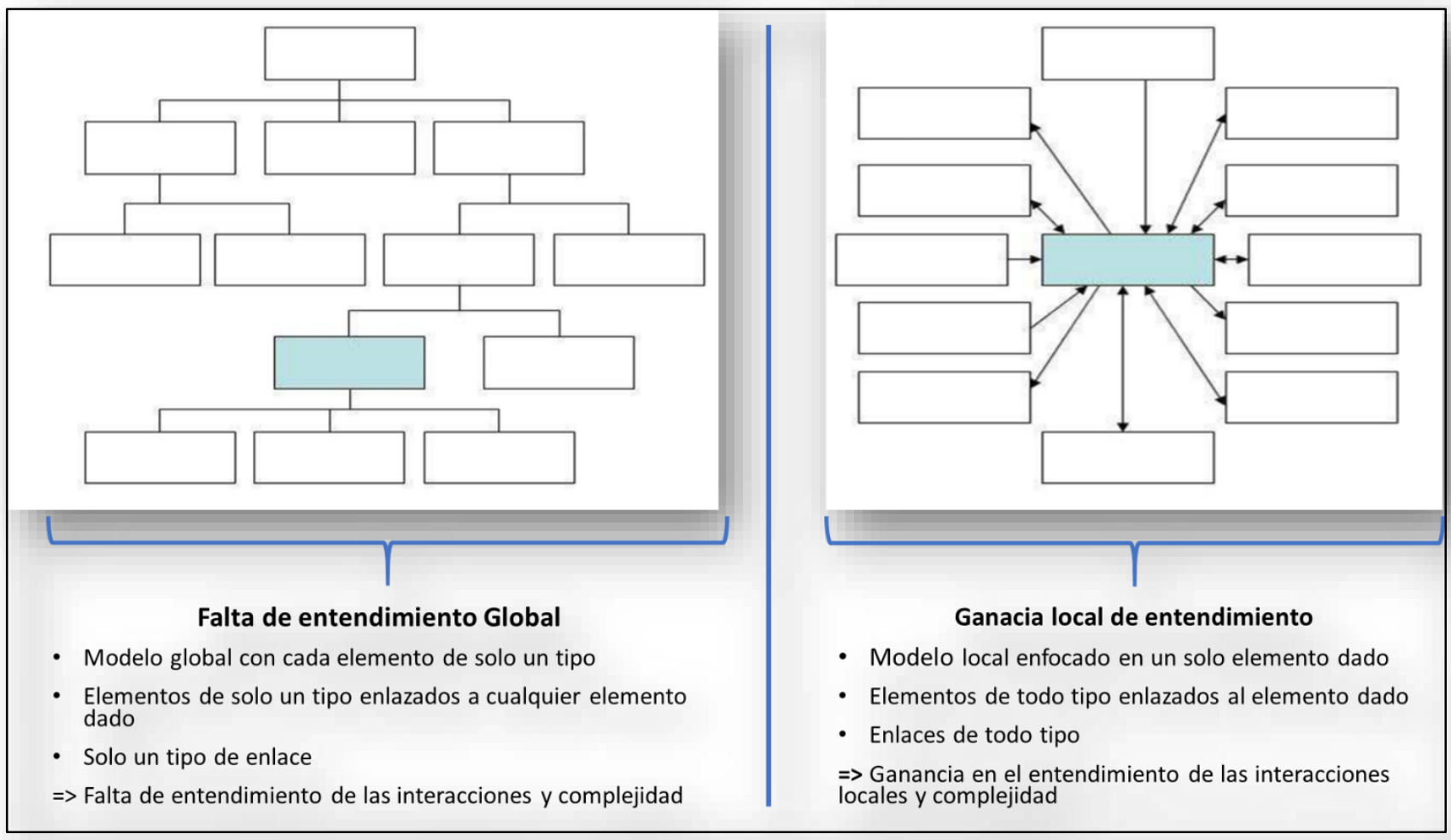

Ilustración 4 - De una falta de entendimiento global a ganancia en el entendimiento local [5] 
Dentro del proceso del modelado existen elementos cualitativos que deben trabajarse:

"Donde a veces habia cierta confusión y falta de acuerdo sobre la definición de todos los elementos que estaban involucrados en un proyecto, ahora bay un marco general y una estructura estandarizada que crea consenso dentro del equipo del proyecto."

\subsubsection{Complejidad vista desde un punto de vista de modelado de categorías, Wood, 2010 [13]}

Wood considera importante tomar en cuenta 16 temas principales para modelar la complejidad de un proyecto y de esta manera definirlo. [13]

Estos temas los subdivide en 5 categorías principales según la siguiente tabla:

\begin{tabular}{ll}
\hline Tema & Sección dentro del tema \\
\hline Organizacional & Resumen del cliente \\
& Estructura Organizacional \\
& El cliente y accionistas del proyecto \\
Planificación y Administración & Coordinación del proyecto \\
& Planificación \\
& Información \\
Operacional y Tecnológico & Tecnología \\
& Nuevos métodos \\
& Dificultad inherente \\
& Tamaño del proyecto \\
Ambiental & Ambiente físico \\
Incertidumbre & Ambiente del proyecto \\
& Localidad \\
& Estructuras existentes \\
& Planificación \\
& Uniformidad \\
\hline
\end{tabular}

Tabla 2- Temas de complejidad de proyectos [13]

La complejidad, incluso menciona las fórmulas previamente establecidas por Nassar and al. [6], pero no aclara cómo las usa en su metodología para modelar la complejidad del proyecto.

\subsubsection{Complejidad de Proyecto utilizando el proceso jerárquico-analítico, Vidal, 2010 [7]}

Similar al acercamiento por Wood [13] y Weaver [8], Ludvic prefiere proponer un método que entra dentro de los métodos holísticos. 
Ludvic utiliza, para su marco de evaluación, 18 factores esenciales repartidas en 4 grandes categorías:

\begin{tabular}{|c|c|c|}
\hline Familia & Complejidad Organizacional & Complejidad Tecnologica \\
\hline Tamaño del sistema del proyecto & 1. Número de accionistas & \\
\hline \multirow[t]{3}{*}{ Variedad del sistema del proyecto } & 2. Variedad de sistemas de informacion a ser combinadas & \\
\hline & $\begin{array}{l}\text { 3. Locación geográfica de los accionistas (y su descontento } \\
\text { mutuo) }\end{array}$ & \\
\hline & 4. Variedad en los intereses de los accionistas & \\
\hline \multirow[t]{10}{*}{$\begin{array}{l}\text { Interdependencias del sistema del } \\
\text { proyecto }\end{array}$} & 5. Dependencias con el ambiente & $\begin{array}{l}\text { 17. Interdependencia de las } \\
\text { especificaciones }\end{array}$ \\
\hline & $\begin{array}{l}\text { 6. Disponibilidad de personas, materiales y de cualquier } \\
\text { otro recurso debido al uso compartido }\end{array}$ & \\
\hline & 7. Interdependecia entre sitios, departamentos y compañías & \\
\hline & $\begin{array}{l}\text { 8. Interconectividad y circuito de retroalimentación en la } \\
\text { tarea y las redes del proyecto }\end{array}$ & \\
\hline & 9. Equipo de cooperación y comunicación & \\
\hline & 10. Dependencias entre cronogramas & \\
\hline & 11. Interdependencia de la informaion de sistemas & \\
\hline & 12. Interdependecia de objetivos & \\
\hline & 13. Nivel de la interrelacion entre fases & \\
\hline & 14. Procesos de interdependencia & \\
\hline \multirow[t]{2}{*}{$\begin{array}{l}\text { Dependencias contextuales del } \\
\text { sistema del proyecto }\end{array}$} & 15. Configuración cultural y variedad & $\begin{array}{l}\text { 18. Complejidad ambiental } \\
\text { (ambiente en red) }\end{array}$ \\
\hline & 16. Complejidad ambiental (ambiente en red) & \\
\hline
\end{tabular}

Tabla 3 - Marco refinado de la complejidad de proyectos [7]

Cabe destacar que Ludvic considera este método una propuesta definitiva para solucionar la definición y medición de la complejidad de un proyecto. Sin embargo, su éxito depende de la opinión de quienes participaron en el estudio mencionado.

Ludvic considera este método:

"fiable, ya que los usuarios finales confían en los resultados, medidas y escalas que se proponen. Durante el estudio de caso, los participantes del caso de estudio nunca impugnaron ningún resultado numérico".

[7]

No hay indicativo en el estudio de la cantidad de personas a las que se refería Ludvic ni existe un estudio estadístico que apoye esta afirmación.

\subsubsection{Complejidad de Proyecto utilizando el método de factores por categorías, Lan, 2016, [9]}

Lan considera que "se cree ampliamente que la complejidad está en gran medida relacionada con la subjetividad del observador". Partiendo de este concepto, coincide, como muchos predecesores, de incluir en su método de medición, aspectos cualitativos en su análisis. La 
siguiente tabla demuestra este acercamiento, con la cual procede a hacer su cálculo de correlación de las respuestas obtenidas por las muestras de campo. Su muestra se basa en 256 encuestados, cuyas respuestas fueron procesadas y categorizadas de la manera mostrada en la tabla siguiente.

Tabla 4 - Factores de complejidad potenciales y medidas de éxito de proyectos complejos de construcción

\begin{tabular}{|c|c|}
\hline Categoría & Factores potenciales \\
\hline Complejidad de la Meta & $\begin{array}{l}\text { Diversidad de objetivos (PC1); incertidumbre de objetivos (PC2); inconsistencia de los objetivos del } \\
\text { proyecto (PC3); cambio de número de requisitos de partes interesadas (PC4); urgencia del proyecto por } \\
\text { límite de tiempo (PC5); urgencia para el costo del proyecto (PC6) }\end{array}$ \\
\hline Complejidad organizacional & $\begin{array}{l}\text { Número de jerarquías de estructura organizacional (PC7); número de unidades organizativas y } \\
\text { departamentos (PC8); interdependencia interorganizacional (PC9); experiencia de los participantes } \\
\text { (PC10); cambio de organización del proyecto (PC11); confianza entre la organización del proyecto } \\
\text { (PC12); sentido de cooperación (PC13); diferencias culturales de organización del proyecto (PC14). }\end{array}$ \\
\hline Complejidad de la Tarea & $\begin{array}{l}\text { Diversidad de tareas (PC15); dependencia de la relación entre tareas (PC16); dinámica de las actividades de } \\
\text { la tarea (PC17); incertidumbre de los métodos y herramientas de gestión de proyectos (PC18); } \\
\text { disponibilidad de recursos y habilidades (PC19); fuentes de financiación (PC20); complejidad de la } \\
\text { relación contractual (PC21) }\end{array}$ \\
\hline Complejidad Tecnologica & $\begin{array}{l}\text { Diversidad de tecnología en proyecto (PC22); dependencia de procesos tecnológicos (PC23); riesgo de } \\
\text { usar tecnología altamente difícil (PC24); conocimiento de nuevas tecnologías (PC25); novedad de } \\
\text { productos de construcción (PC26) }\end{array}$ \\
\hline Complejidad Ambiental & $\begin{array}{l}\text { Entorno de cambio de políticas y regulación (PC27); entorno de cambio de economía (PC28); entorno de } \\
\text { cambio de naturaleza (PC29); condiciones geológicas complicadas (PC30); cambios en el entorno de } \\
\text { construcción del proyecto (PC31); lejanía de la ubicación del proyecto (PC32); influencia de las partes } \\
\text { interesadas externas (PC33) }\end{array}$ \\
\hline $\begin{array}{l}\text { Complejidad de la } \\
\text { Informacion }\end{array}$ & $\begin{array}{l}\text { Incertidumbre de la información (PC34); nivel de procesamiento de información (PC35); capacidad de } \\
\text { transferir información (PC36); grado de obtención de información (PC37); integración de más de un } \\
\text { sistema o plataforma (PC38); dependencia del sistema de información (PC39); variedad de lenguaje } \\
\text { involucrado (PC40); Número de países o nacionalidades involucrados (PC41) }\end{array}$ \\
\hline Éxito del Proyecto & $\begin{array}{l}\text { Tiempo (PS1); costo (PS2); calidad (PS3); salud y seguridad (PS4); desempeño ambiental (PS5); } \\
\text { satisfacción de los participantes (PS6); satisfacción del usuario (PS7); valor comercial (PS8) }\end{array}$ \\
\hline
\end{tabular}

\subsubsection{Conclusiones sobre las metodologías de la vertiente holística}

La vertiente holística/cualitativa sopesa o pondera los factores cualitativos para tratar de cuantificar elementos que, de otra manera, serían muy difíciles de medir. En estos casos, el proponente, se ve en la difícil tarea de formular un método que a todas luces tiene aspectos subjetivos, porque las ponderaciones se hacen en base a la experiencia u opinión del autor, o que está avalada por la opinión de un grupo de personas de manera más o menos democrática.

Una cantidad muy pequeña de la literatura relacionada con esta temática abarca métodos cuantificables para la obtención de un método de medición de la complejidad de proyectos. Estos métodos se basan normalmente en la teoría de grafos aplicada, y la mayoría de las veces participan los nodos, arcos, métodos gráficos de simplificación y, en algunos casos, una combinación de los factores mencionados. Algunos métodos incluyen recursos, duraciones de las actividades y otros elementos que no son información de fácil acceso en todos los proyectos, y por ende de estos no se puede realizar una generalización aplicable. 
La literatura más reciente continúa inclinándose por un enfoque holístico, dejando para la década pasada aquellas investigaciones relacionadas al modelado del cronograma, siendo esta última vertiente la que produce figuras de índices de complejidad más intuitivas y sencillas que prometen su facilidad de uso, en la profesión de gestión de proyectos. Sin embargo, carecen de una explicación convincente de su utilidad basándose en los conceptos de cronogramas de proyectos y cuáles son sus funciones dentro de un proyecto.

Reafirmamos que es posible seguir por la vertiente del modelado de cronogramas propuesto para mostrar que una teoría de grafos ayuda en cierta medida, pero no completamente, a la medición de la complejidad del proyecto en sí, tratando de seguir algunos métodos por su sencillez de uso, sin dejar de ser cuantificable.

\subsection{Complejidad de proyectos - vertiente del modelado de cronogramas}

Una cantidad muy pequeña de la literatura relacionada con esta temática se centra en la obtención de un método de medición de la complejidad de proyectos [6,23,24,25] basándose en modelos de los proyectos con información "concreta". Estos métodos se basan generalmente en la teoría de grafos aplicada, y participan la mayoría de las veces los nodos y arcos de la red del cronograma de proyectos, con métodos que incluyen la simplificación de la red, y algunos de ellos, una combinación de lo anterior. Algunos métodos incluyen recursos, duraciones de las actividades y otros elementos que no son información de fácil acceso en todos los proyectos, y por ende de estos no se puede realizar una generalización aplicable [6].

La discusión que produce este método resulta en dos formas de definición de complejidad. Nassar y Hegab definen su método como "la medición de la complejidad del cronograma del proyecto" [23], o sea la medición de complejidad de un aspecto del total de la complejidad de un proyecto, mientras que Vidal et al. definen este método como "las mediciones del modelo de la estructura del proyecto representada como un grafo" $[7,26]$. Ninguno percibe este acercamiento como algo que logre definir la complejidad de un proyecto en su totalidad.

Aquí es donde la definición de un proyecto y de complejidad deben ayudar a definir justamente el alcance de estos métodos y mediciones. Al no haber un consenso de este, se presenta la siguiente síntesis para obtener una definición base de esta investigación:

1. Un proyecto se define, según el PMBOK, como el esfuerzo que se lleva a cabo para crear un producto, servicio o resultado único, y tiene la característica de ser temporal. No es ni el producto, servicio o resultado único por lo cual se hacen estos esfuerzos [1].

2. "Un sistema es un objeto, que, en un ambiente dado, intenta alcanzar un objetivo (aspecto teleológico) al realizar una actividad (aspecto funcional) mientras su estructura interna (aspecto ontológico) evoluciona con el tiempo (aspecto genético) sin perder su identidad" [7].

3. Y que "la complejidad de un proyecto es la propiedad de un proyecto que hace difícil comprender, prever y mantener en control su comportamiento general, aun cuando se 
haya dado información razonablemente completa sobre el sistema del proyecto” [7].

Tomando en cuenta estos 3 conceptos, se aprecia la utilidad del modelado de un cronograma de obra que abarque los siguientes elementos:

1. Se establece la estructura del proyecto (aspecto ontológico)

2. Se establece el objetivo final, incluso objetivos parciales (aspecto teleológico)

3. Se modela los esfuerzos por medio de actividades (aspecto funcional)

4. Se modela su evolución con el tiempo (aspecto genético)

5. Sus elementos están interrelacionados para tener secuencia, unidad, e identidad.

6. Contiene, si está bien realizado, la principal y mayor cantidad de información necesaria para poder administrar un proyecto.

De lo anterior, se considera que medir la complejidad de un cronograma es lo más cercano a medir, por definición, la complejidad de un proyecto.

Otros elementos externos serian meta-proyectuales o multi-proyectuales (más de un proyecto), y aunque son útiles, no serán fundamento de esta investigación.

\subsubsection{Metodologías de análisis de complejidad de proyectos por la vertiente del modelado de cronogramas}

Las fórmulas propuestas que están relacionadas con el tema de la complejidad de proyectos calculado en base a los cronogramas de obras se presentan aquí de forma cronológica según la fecha de los artículos de referencia.

Antes de continuar, la historia de estos métodos está directamente relacionada a la evolución que hubo en el desarrollo del método de creación de redes de cronogramas de proyectos. En un inicio se basaban mucho en las redes en donde las actividades eran representadas en los arcos o flechas de las redes (ver ilustración 5). Posteriormente se adoptó el método de representar las actividades en los nodos (ver ilustración 6).

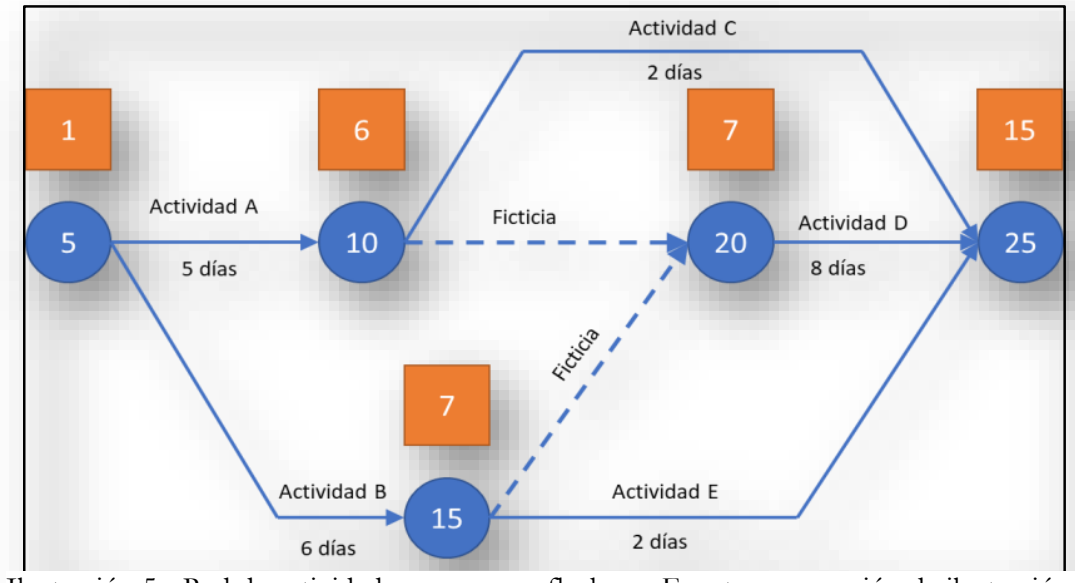

Ilustración 5 - Red de actividades en arcos o flechas - Fuente - recreación de ilustración encontrada en https://knowledge4civil.wordpress.com/2017/04/09/uses-of-cpm-andpert-in-construction-management/ 


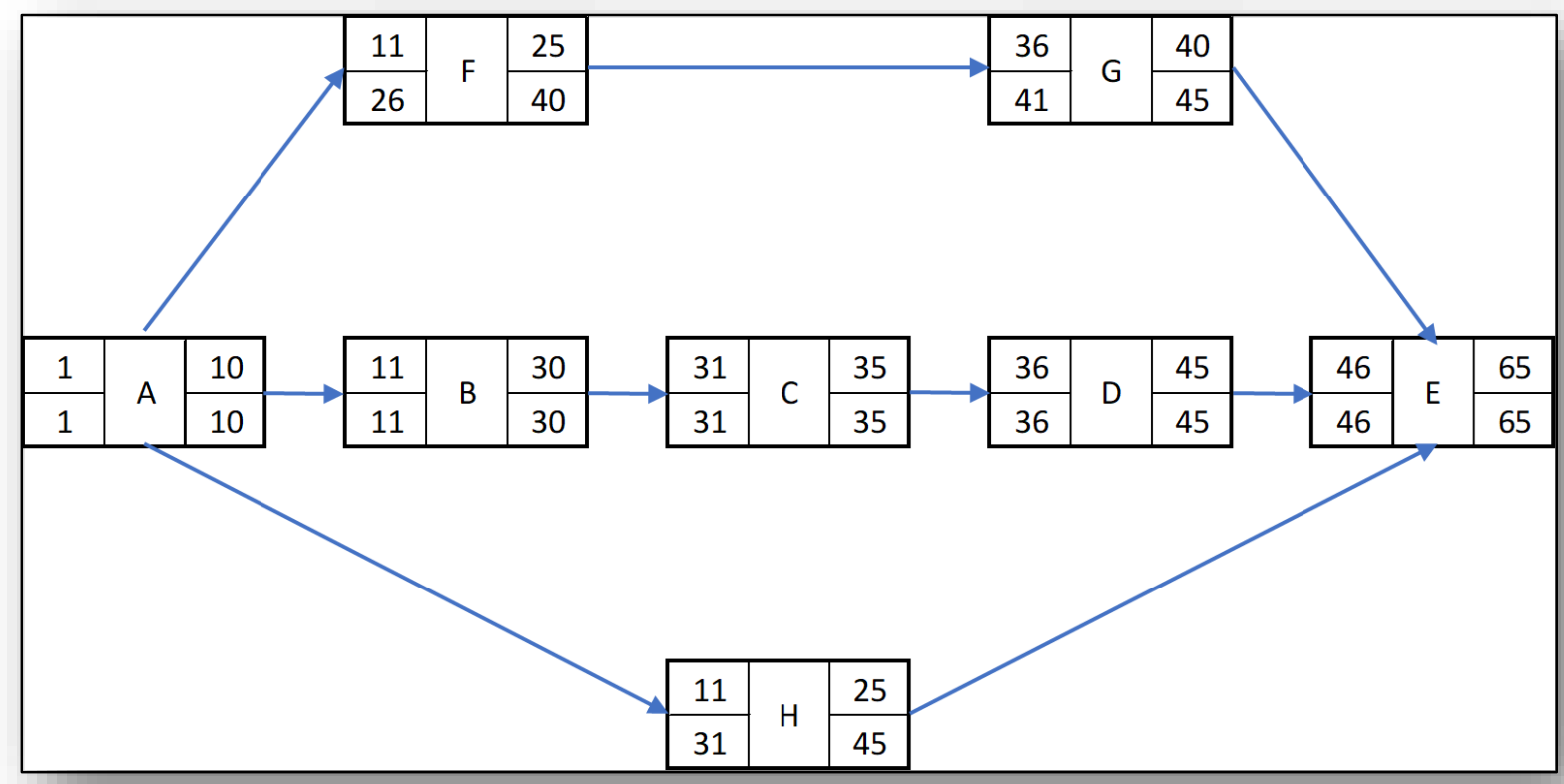

Ilustración 6 - Red de actividades en nodos - fuente: simplificación de ilustración encontrada en https://www.tomfanelli.com/how-to-visualize-your-infographic-part-3/

\subsubsection{Pascoe, 1966, Red de Actividad en Flecha}

La fórmula de Pascoe resulta en índice simple, en la cual solo se toman, sin modificaciones o análisis previos, el número de actividades y el número de arcos de un cronograma.

$$
C=a / n
$$

En donde "C" es el índice de complejidad de proyectos, "a" el número de relaciones o arcos, y " $\mathrm{n}$ ” el número de nodos o actividades. [6]

\subsubsection{Davies, 1974, AOA}

Davies, ya utilizando teoría de grafos, reconoce que existe un límite máximo y un límite mínimo de relaciones posibles por número de nodos (actividades) en una red. A esto le da un factor de 2x de forma arbitraria para ayudar al cálculo del análisis.

$$
C=(2 x(a-n+1)) /((n-1)(n-2)
$$

En donde "C" es el índice de complejidad de proyectos, "a" el número de relaciones o arcos, y "n" el número de nodos o actividades. [6]

\subsubsection{Kaiman, 1974, redes AOA}

Kaiman resuelve su fórmula de manera similar a Pascoe, solo agregándole un factor al cuadrado al número de arcos/relaciones. 


$$
C=\left(a^{2}\right) /(n)
$$

En donde "C" es el índice de complejidad de proyectos, "a" el número de relaciones o arcos, y " $\mathrm{n}$ ” el número de nodos o actividades. [6]

\subsubsection{Kaiman, 1974, redes AON (pero no toma en cuenta los nodos).}

Con esta fórmula, Kaiman ignora los arcos y contempla solo las actividades, utilizando el número de actividades precedentes para establecer una proporcionalidad con el número de actividades totales.

$$
C=\left(a_{p}^{2}\right) /(a)
$$

En donde " $a_{p}$ " es el número de actividades precedentes y " $a$ ” el número de actividades [6], enfocándose más en las situaciones de embudo que pueden suceder en la consecución de actividades en un cronograma.

\subsubsection{Badiru citado por Nassar et al., junio 2006}

Badiru presenta una fórmula del índice de complejidad de proyectos tomando en cuenta más variables que solo actividades y relaciones de una red del cronograma. La fórmula es la siguiente:

$$
C=\frac{p}{d}\left[\left(1-\frac{1}{L}\right) \times \sum_{i=1}^{L} t_{i}+\sum_{j=1}^{R}\left(\frac{\sum_{i=1}^{L} t_{i} x_{i j}}{Z_{j}}\right)\right]
$$

"Donde $L=$ numero de actividades en la red; $t i=$ duración esperada de la actividad $i ; \mathrm{R}=$ numero de tipos de recursos; xij =unidades de los recursos tipo j requeridas por la actividad $i ; \mathrm{Zj}=$ número máximo del tipo de recursos $j$ disponibles; $p=$ máximo número de predecesores inmediatos en la red; $y d=$ la duración PERT del proyecto sin restricciones de recursos.”. [6]

Esta es una fórmula orientada a medir la complejidad de una sola actividad, y tendría que hacerse interacciones de esta por cada actividad para poder evaluar la complejidad de un proyecto completo. Tiene la dificultad de que no muchos cronogramas incluyen en todas sus actividades recursos específicos a la tarea. Por lo cual en algunos proyectos el índice total del proyecto resulta parcialmente incorrecto. 


\subsubsection{Medida de complejidad para cronogramas de proyectos, Khaled M. Nassar1 and Mohamed Y. Hegab, Junio 2006, AON [6]}

$$
C_{n}= \begin{cases} & \left\{\frac{\log \left[\frac{a}{n-1}\right]}{\log \left[\frac{n^{2}-1}{4(n-1)}\right]}\right\} \% \text { si } n \text { es impar } \\ & \left\{\frac{\log \left[\frac{a}{n-1}\right]}{\log \left[\frac{n^{2}}{4(n-1)}\right]}\right\} \% \text { si } n \text { es par }\end{cases}
$$

En donde " $a$ "representa los arcos y " $n$ " los nodos o actividades. Esta fórmula no se asegura que los dígrafos no sean mínimos, pero si contempla los límites como aquellos que tengan arcos de redundancia. También utiliza dígrafos que tengan un número máximo de arcos como base para una banda superior de medición. [6] Cabe mencionar que dentro de estos análisis no se contemplan proyectos en que las últimas actividades no estén relacionadas entre sí, tema que se abarca dentro de la propuesta al proponer un hito final como un estándar de desarrollo de cronogramas.

\subsection{8. Índice de Complejidad de Proyectos basado en la Topología, Boushaala, 2010 [25]}

Boushala propone aumentar el detalle de los factores a variables a utilizar a la hora de realizar la métrica de la complejidad del proyecto, agregando las rutas críticas a las variables de número de actividades y relaciones, ya establecidas.

$$
\begin{aligned}
& \operatorname{CNC}(P R 1)=\left[\frac{W}{1-\frac{A C}{A}}\right]\left[\frac{P}{C P}\left\{\left(1 \frac{1}{A}\right) \sum_{i=1}^{A} t i+\sum_{j=1}^{R}\left(\frac{\sum^{A} t i r_{i j}}{R A_{j}}\right)\right\}\right] \\
& \operatorname{CNC}(P R 2)=A C / A \\
& \operatorname{CNC}(P R 3)=\left[\frac{1}{1-\frac{A C}{A}}\right]\left[\frac{P}{C P}\left\{\left(1 \frac{1}{A}\right) \sum_{i=1}^{A} t i+\sum_{j=1}^{R}\left(\frac{\sum^{A} t i r_{i j}}{R A_{j}}\right)\right\}\right]
\end{aligned}
$$

Dónde:

$\boldsymbol{W}=$ número de rutas críticas en la red

$\boldsymbol{A} \boldsymbol{c}=$ número de actividades críticas en la red

$\boldsymbol{A}=$ número de actividades en la red 


\subsubsection{Basado en la centralidad y centralidad de rutas. Khan, 2010 [27]}

Para Khan, los índices propuestos anteriormente no representan una complejidad del proyecto o cronograma, sino una medición de la calidad de esta [27]. Critica los métodos anteriores por no incluir suficientes factores de medición, como la duración de las actividades. Afirma que solo funcionan para comparar un proyecto con otra instancia de sí misma, y que la definición de complejidad está limitada a la relación entre número de arcos y actividades [27].

Sin embargo, Khan no llega a medir complejidad, si no la estabilidad misma del cronograma, definiendo su índice de centralidad como la medición de la buena calidad de un cronograma que a su vez sucede cuando los valores de la ruta crítica son estables y no es seriamente afectada por cambios menores en la duración de una actividad [27]

Khan presenta la siguiente formula:

$$
\text { Valor de Centralidad }=C v=\left[100 \times \frac{1}{|N|} \sum_{n e N}\left[\frac{\max (n)}{C} d(\rho)\right]\right] \%
$$

$$
\text { Centralidad de Rutas }=C p=\left[100 \times \frac{\mid\{n: n \text { no es una actividad critica }\} \mid}{|N|}\right] \%
$$

Aquí "N" denota el número de nodos en el grupo " $N "$ y " $\max _{\rho e P(n)} d(\rho)$ " es la duración de la ruta de abismo (sink path) a través del nodo "n”. Estas definiciones fueron inspiradas por el análisis de redes en donde el concepto de centralidad fue utilizado para identificar los actores más importantes (Bataglj, 1996, Brandes, 2001). Khan interpreta la medida de calidad de forma similar, definiendo cantidad como:

$$
\text { Rango del Nodo }=R(n)=\frac{\max _{p e P(n)} d(\rho)}{C}
$$

1.4.10. Método para la eliminación de redundancias en una red, H.A. Bashir, Julio 2010 [24]

Este método se presenta aquí como un elemento importante, tal como su creador indica, como soporte al método de Nassar, presentado en el punto 1.2.7. Este método provee la manera de reducir los arcos de una red a un mínimo. 


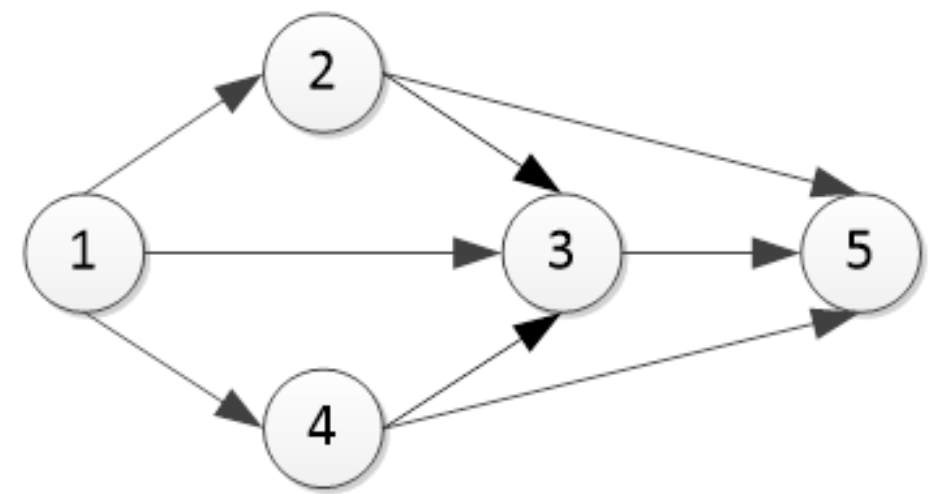

Ilustración 7 - Red de actividades en nodos del proyecto hipotético [24]

1.4.10.1. Basándose en una matriz de red de actividades como la red y la matriz ejemplificada arriba, el método consiste en los siguientes pasos:

1. Construcción de la matriz de predecesores inmediatos, MIP (Matrix of Inmediate Predecessors);

\begin{tabular}{|c|c|c|c|c|c|}
\hline Tabla 1. & \multicolumn{5}{|c|}{ Matriz de Predecesores Inmediatos del Proyecto Hipotetico } \\
\hline & \multicolumn{5}{|c|}{ Actividad } \\
\hline Actividad & 1 & 2 & 3 & 4 & 5 \\
\hline 1 & 1 & 1 & 1 & 1 & 0 \\
\hline 2 & 0 & 1 & 1 & 0 & 1 \\
\hline 3 & 0 & 0 & 1 & 0 & 1 \\
\hline 4 & 0 & 0 & 1 & 1 & 1 \\
\hline 5 & 0 & 0 & 0 & 0 & 1 \\
\hline
\end{tabular}

Tabla 5 - Matriz de predecesores inmediatos del proyecto hipotético [24]

2. La transformación del MIP a una matriz de formato de menor nivel de formato triangular, o LTF (Lower Triangular Format Matrix);

\begin{tabular}{|c|c|c|c|c|c|}
\hline Tabla 2. & \multicolumn{5}{|c|}{ Matriz Triangular Inferior del Proyecto Hipotético } \\
\hline & \multicolumn{5}{|c|}{ Actividad } \\
\hline Actividad & 5 & 3 & 2 & 4 & 1 \\
\hline 5 & 1 & 0 & 0 & 0 & 0 \\
\hline 3 & 1 & 1 & 0 & 0 & 0 \\
\hline 2 & 1 & 1 & 1 & 0 & 0 \\
\hline 4 & 1 & 1 & 0 & 1 & 0 \\
\hline 1 & 0 & 1 & 1 & 1 & 1 \\
\hline
\end{tabular}


3. Transformación del LTF en una matriz de mínimos arcos adyacentes;

4. La construcción del dígrafo de mínimos arcos.

1.4.10.2. El método para transformar una LTF en una Matriz de mínimos arcos es la siguiente:

1. Reemplazar todas las entradas diagonales por " 0 ".

2. Después de transformar todas las entradas diagonales de la LTF de "1" "a "0" s, cada columna es consultada en secuencia, desde la columna 1 a la columna 5, para identificar las entradas de " 1 ".

3. La búsqueda nos debe mostrar que la primera entrada que tiene valor de " 1 " es la entrada e21 (la entrada en la columna 2 y fila 1).

4. Se procede a buscar en la fila 2 por celdas que contengan " 1 " que estén en las columnas mayores de 2, y las entradas correspondientes en la fila 1, e31, e41, e51, son restringidas a 0 , siempre y cuando la fila 2 tenga el valor de " 1 " en esas columnas.

5. Continuar el proceso anterior resulta en la matriz de mínimos arcos adyacentes, mostrada en la Tabla

\begin{tabular}{|c|c|c|c|c|c|}
\hline Tabla 3. & \multicolumn{5}{|c|}{ Matriz de Mínimos Arcos Adyacentes del Proyecto Hipotético } \\
\hline & \multicolumn{5}{|c|}{ Actividad } \\
\hline Actividad & 5 & 3 & 2 & 4 & 1 \\
\hline 5 & 0 & 0 & 0 & 0 & 0 \\
\hline 3 & 1 & 0 & 0 & 0 & 0 \\
\hline 2 & 0 & 1 & 0 & 0 & 0 \\
\hline 4 & 0 & 1 & 0 & 0 & 0 \\
\hline 1 & 0 & 0 & 1 & 1 & 0 \\
\hline
\end{tabular}

Tabla 7 - Matriz de mínimos arcos adyacentes del proyecto hipotético [24]

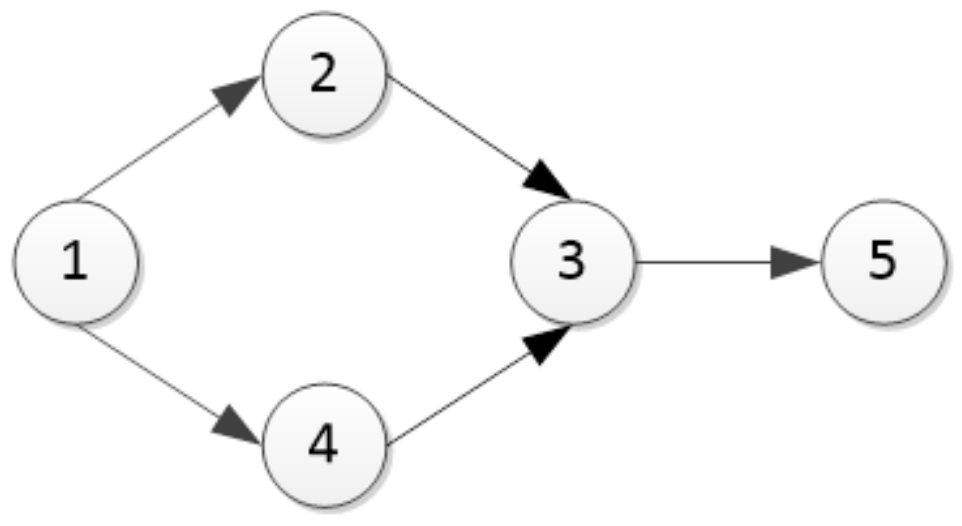

Ilustración 8 - Red de mínimos arcos del proyecto hipotético [24] 


\subsection{Complejidad vs Desempeño}

No hay un estudio comparativo que haga una correlación entre los índices de complejidad desarrollados por el método de modelado de grafos y el desempeño de los proyectos. Por ende, es difícil (con la documentación actual), evaluar si la complejidad definida en estos métodos realmente describe la complejidad de proyectos como un factor determinante en la ejecución de un proyecto, aunque todos están de acuerdo en que sí. La literatura actual se dedica a encontrar un método utilizable mas no hace la comparación de estos métodos o índices propuestos con el desempeño de proyectos para así tener una estadística que permita observar esta relación. Por ende, parte de esta investigación es proveer tal estadística.

Sin embargo, dos autores han hecho la correlación entre la complejidad utilizando el método holístico y el desempeño o éxito de un proyecto. Lan [9] y Yang [28] resuelven hacer el estudio de la relación tomando en cuenta el éxito de un proyecto, no el desempeño del mismo. Lan, comprende el desempeño del proyecto como un factor incluido dentro del concepto de éxito, o sea como uno de los factores a tomar en cuenta dentro de todas sus categorías. Yang, por su parte, no toma en cuenta el desempeño como índice métrico, sino como parte de su estudio comparativo entre el buen liderazgo y el éxito del proyecto.

Parte del valor de esta investigación es poder realizar la correlación entre dos índices razonables de conseguir e intuitivos y observar si puede ser aplicado de forma regular al análisis de proyectos a futuro.

\subsection{Conclusiones}

Durante la revisión de estos métodos se ha observado que algunos de ellos han dejado de ser de mayor utilidad. Todos los métodos que basan sus redes de cronograma en actividades en arcos son obsoletos debido a que hoy todos los cronogramas se basan en redes en donde las actividades se representan por nodos.

Otros métodos incluyen elementos como recursos o duraciones de las actividades para definir complejidad. Los recursos en un cronograma no siempre pueden utilizarse para la evaluación de un cronograma porque no siempre las actividades están cargadas con recursos, incluso en algunos casos no es práctico utilizar recursos en un cronograma, aunque sea lo ideal o deseable.

Las duraciones de las actividades podrían ser un elemento a tomar en cuenta, sin embargo, los métodos propuestos, como en el propuesto por Kahn [27] o Boushala [25], usan como base el concepto de que a medida que un proyecto se atrasa, empieza a incrementar la cantidad de rutas casi-críticas, por ende aumenta la cantidad de actividades concurrentes que deben realizarse, y más actividades en la cual la gestión de proyectos debe enfocarse de forma concurrente.

Este concepto igual va de la mano con el concepto de redes y arcos, sin necesidad de un análisis de la criticalidad misma, ya que la criticalidad no es en sí un factor de complejidad. Una red lineal tiene todas sus actividades críticas. Lo que hace más complejo un proyecto parece ser la cantidad de actividades a la cual el gerente debe poner atención al mismo tiempo. Un análisis estrictamente de arcos y nodos se acerca más a este paralelismo y por ende funciona como índice de análisis de la complejidad de proyectos. Se debe crear un índice de paralelismo del cronograma en adición a la proporcionalidad de arcos y nodos, lo cual será tema de futuras investigaciones. 


\subsection{Referencias}

[1] Project Management Body of Knowledge (PMBOK Guide), Project Management Institute. Newtown Square, Pa., 2008, 4th Ed.

[2] Baccarini, David. "The concept of project complexity-a review," International Journal of Project Management, Vol. 14, No. 4, pp.201-204, (1996).

[3] Wood, Hannah Louise, Ashton, Philip. "The Factors of Project Complexity," 18th CIB World Building Congress. Salford, UK., 2010

[4] Frame, J. Davidson. The New Project Management, Tools for an Age of Rapid Change, Complexity, and other Business Realities, Jossey-Bass A Wiley Company, San Francisco, Ca., 2nd Ed. 2002

[5] Vidal, Ludovic-Alexandre, Marle, Franck, Bocquet, Jean-Claude. "Modelling Project Complexity," ICED’07/515, 2007.

[6] Nassar, Khaled M., Hegab, Mohamed Y. "Developing a Complexity Measure for Project Schedules," Journal of Construction Engineering and Management, Vol. 132, No. 6, June 2006.

[7] Vidal, Ludovic-Alexandre, Marle, Franck, Bocquet, Jean-Claude. "Measuring Project Complexity using the Analytic Hierarchy Process," International Journal of Project Management 29 pp.718-727, 2011

[8] Weaver, Patrick. "Scheduling in the Age of Complexity," PMI College of Scheduling Annual Conference 17 - 20 May 2009

[9] Luo, Lan, et. al. "Investigating the Relationship between Project Complexity and Success in Complex Construction Projects," Journal of Management in Engineering, July 2016

[10] Fostel, C. (2011). The value of earned value management. Paper presented at PMI@ Global Congress 2011—North America, Dallas, TX. Newtown Square, PA: Project Management Institute." [Online]. Available: https://www.pmi.org/learning/library/value-earned-value-management-6232

[11] Reichel, C. W. (2006). Earned value management systems (EVMS): "you too can do earned value management" Paper presented at PMI® Global Congress 2006-North America, Seattle, WA. Newtown Square, PA: Project Management Institute [Online]. Available: pmi.org/learning/library/earned-value-management-systems-analysis-8026

[12] Real Academia Española [Online]. Available: dle.rae.es/holismo?m=form; dle.rae.es $/ \mathrm{hol} \% \mathrm{C} 3 \% \mathrm{AD}$ stico

[13] Wood, Hannah Louise, Ashton, Philip. "Modelling Project Complexity,” Procs 26th Annual ARCOM Conference, pp.1111-1120, September 2010

[14] Xia, Bo \& Chan, Albert. "Measuring Complexity for Building Projects-A Delphi Study," Engineering, Construction and Architectural Management Vol. 19 No. 1, pp. 7-24, 2012

[15] Akilesawaran, V, Hazez, G.B., Morin, T.L. "Complexity of the Project Sequencing Problem," 
Operations Research · August 1983

[16] Castejón-Limas, Manuel, Ordieres-Meré, Joaquín, González-Marcos, Ana, González-Castro, Víctor. "Effort estimates through project complexity," Springer Science+Business Media, LLC [Online]. Available:

www.researchgate.net/publication/220461783 Effort estimates through project complexity Jul. 16, 2010].

[17] Henriksen, JO “Taming the Complexity Dragon," Winter Simulation Conference - Titans of Simulation, 2006.

[18] Svetlana Cicmil, Terry Cooke-Davies, Lynn Crawford, and Kurt Richardson. Exploring the Complexity of Projects. Project Management Institute. Newtown Square, Pa, Kindle Edition, 2009.

[19] Obolensky, Nick. Leading Complex Projects. ICCPM. Kindle Ed., 2013.

[20] Terry, Cooke-Davies. Aspects of Complexity: Managing Projects in a Complex World. Project Management Institute. Newtown Square, Pa. Kindle Ed., 2011

[21] Cavanagh, Michael. Project Complexity Assessment. ICCPM. Kindle Ed., 2013.

[22] Sinha, Sanjeev. "An Innovative Technique to Measure Complexity Of A Construction Project Activity,” Architectural Engineering and Design Management · January 2006

[23] Nassar, Khaled. "Schedule Network complexity vs Project complexity," Proceedings of the International Conference on Computing in Civil and Building Engineering (2010).

[24] Bashir, H. A. "Removal of Redundant Relationships in an AON Project Network for Evaluating Schedule Complexity," Journal of Construction Engineering and Management (C) ASCE pp.787 -793, July 2010.

[25] Boushaala, Amer A. "Project Complexity Indices based on Topology Features," World Academy of Science, Engineering and Technology 69, pp. 49-54, 2010.

[26] Trudeau, Richard J. Introduction to Graph Theory. Dover Publications Inc., New York, 19932 ed.

[27] Khan, Muhammad Ali. "Quantitative quality assessment of network-based schedules" Proceedings of the International Conference on Computing in Civil and Building Engineering, 2010.

[28] Yang, Li-Ren et al. “The association among project manager's leadership style, teamwork and project success” International Journal of Project Management, 2011 